\title{
REFLEXIONES SOBRE EL USO DE LAS INSTITUCIONES DE MICROFINANZAS COMO INSTRUMENTOS DE DISPERSIÓN DEL CRÉDITO PÚBLICO A MICRONEGOCIOS
}

\author{
Luis Alfredo Castillo Polanco \\ Doctor en Economía y Profesor de Tiempo Completo \\ de la Universidad de Quintana Roo.
}

Edgar Alfonso Sansores Guerrero Maestro en Economía y Administración Pública y Profesor de Tiempo Completo de la Universidad de Quintana Roo. 



\section{I.- INTRODUCCIÓN}

Tradicionalmente la intervención del gobierno en la economía ha sido a través del marco regulatorio, la implementación de la política fiscal y económica; y la proveeduría de bienes públicos. Sin embargo, su participación en la asignación de fondos públicos a instituciones microfinancieras resulta relativamente nueva y controversial.

Las organizaciones impulsoras y destacados analistas han llegado a la conclusión de que la participación del Gobierno en el microfinanciamiento no es deseable ${ }^{1}$. En México, sin embargo, el gobierno participa, no como oferente de microcrédito al menudeo, pero si financiando a las propias instituciones de microcrédito. Con esta política el Gobierno puede verse como un mayorista que utiliza a las Instituciones de Microfinanzas (IMF's) como entidades dispersoras del crédito público.

El presente artículo explora las posibles implicaciones que tiene la intervención del gobierno en el mercado del microcrédito a través del financiamiento a IMF's: la sostenibilidad de las IMF (cumplimiento y tasas que recuperen los costos de proporcionar el crédito), la eficiencia y fomento a la competencia, el alcance e impacto; así como en la democratización del crédito. Este documento pretende contribuir a la discusión sobre la participación de Estado en el mercado crediticio, especificamente en el financimiento a IMF’S en México.

Para el logro del objetivo de la investigación se analiza el Programa Nacional de Financiamiento al Microempresario (PRONAFIM), el cual, al utilizar a organizaciones de microfinanzas privadas como dispersoras de crédito, representa una experiencia novedosa como programa público para el combate contra la pobreza y la exclusión de las actividades económicas de varios sectores de la población, tanto por su oferta de financiamiento público a IMF's, como por su propósito de brindar servicios financieros y asistencia técnica con una visión integral de las necesidades de la sociedad.

\section{II.- LA CRÍTICA AL FINANCIAMIENTO PÚBLICO Y LAS MICROFI- NANZAS}

En su texto "La Economía de las Microfinanzas", Armendáriz de Aghion y Morduch (2007), afirman que es el repudio al legado negativo que ha dejado el desempeño del sector público como oferente de financiamiento lo que ha orientado al movimiento de las microfinanzas al sector privado en busca de inspiración.

${ }^{1}$ Sus argumentos se discuten en el siguiente apartado. 
Por ello, el CGAP (Grupo Consultivo para la Asistencia a los pobres), organismo de Banco mundial encargado de la promoción, estudio y definición de políticas y lineamientos para las microfinanzas, en su principio número 8 enuncia "El papel del gobierno es uno de facilitador, no el de proveedor directo de servicios"(CGAP, 2004).

La argumentación de los participantes en el Rural Finance Program de Ohio State University (OSU) (Adams et al, 1987), en torno a los resultados financiamiento público en el sector rural se considera el detonador de esta crítica. La argumentación de OSU específica en torno a los efectos del financiamiento público en el sector rural se basa en la perspectiva teórica más amplia ofrecida por teoría de la represión financiera (Shaw y Mckinnon, 1973); también llamada neoliberalismo (Galviz, 1981).

\section{La eficiencia}

Mckinnon (1973) y Shaw (1973) afirman que la intervención del gobierno en los mercados financieros es indesaeable. Su argumentación se orienta en las políticas aplicadas en Latinoamérica durante las décadas de 1950's y 6o's del siglo pasado. El argumento central es que los gobiernos de aquel entonces se esforzaban por recoger fondos del sistema financiero a través de controles como el encaje legal, bancos de propiedad gubernamental y con la emisión inflacionaria de dinero; y que, bajo la percepción errónea de que una baja tasa de interés impulsa el crecimiento económico, los gobiernos de aquel entonces, a veces denominados "populistas", imponían techos a la tasa de interés.

De acuerdo a la teoría de la represión financiera, las intervenciones estatales que pretendían ampliar la oferta de financiamiento otorgando crédito a precios subsidiados (bajas tasas de interés) a través de organizaciones financieras públicas no lograban su propósito. Por el contrario, no sólo no incrementaban la oferta de crédito, sino que, al desestimular el ahorro con tasas de interés muy bajas, la reducía. Adicionalmente, el fijar las tasas de interés distorsiona las señales de mercado, por lo que esta política de crédito "barato" reducía la eficiencia en la asignación de los recursos (los recursos financieros no eran orientados hacia los mejores proyectos). La perspectiva neoliberal afirma que si el mercado pudiera operar libremente entonces los recursos se canalizarían hacia los proyectos más eficientes, esto es, aquellos que pudieran pagar las mayores tasas de interés. Complementariamente, las altas tasas de interés de mercado inducirían mayor ahorro permitiendo incrementar la profundización financiera y el crecimiento económico en general. En resumen, la "Teoría de la Represión Financiera" afirma que si el capital es escaso, las altas tasas de interés son deseables. 


\section{La critica de Ohio State University (OSU) (Rural finance program}

La crítica de OSU (Dale et al, 1987), afín a la perspectiva de Shaw y de McKinnon, ha mostrado la posibilidad de que la provisión de servicios financieros por parte financieras estatales haya generado distorsiones que lejos de ayudar a combatir la pobreza la habrían acentuado. En general, la crítica al financiamiento público indica que éste habría generado sesgos improductivos, al no favorecer a los proyectos más rentables, e inequidad al asignar crédito mediante criterios políticos.

La crítica de OSU nos ofrece interesantes criterios con los cuales podemos analizar la intervención estatal en el mercado del financiamiento. González (1987) menciona que debido a que la oferta del gobierno consiste en créditos subsidiados, la asignación de estos subsidios se realizará con criterios ajenos a los de mercado, (los favorecidos generalmente son aquellos con influencia o capacidad de presión sobre el gobierno). Cuando las condiciones financieras se agudizan y las bajas tasas significan un subsidio mayor, el monto de crédito subsidiado asignado a los privilegiados crecerá; la brecha en el acceso a financiamiento entre los sectores privilegiados y los no privilegiados crecerá. González considera este resultado tan regular que lo denomina "ley de hierro del financiamiento público".

La crítica de OSU afirma que la asignación administrativa del crédito induce la redistribución del ingreso, pero esta es regresiva. Las bajas tasas de interés no solo desestimulan el ahorro y, en consecuencia, provocaran futuras alzas en este precio mañana cuando el ahorro escasee, sino que al no cubrir los costos de operación harán que el proceso de otorgamiento del crédito sea no sostenible. La no sostenibilidad del crédito, bajo una economía financieramente reprimida o con crédito estatal subsidiado, es uno de los argumentos centrales del enfoque de las microfinanzas.

La interferencia estatal en la asignación del crédito ha generado otro de los problemas centrales con los que pretende lidiar el enfoque de las microfinanzas: el incumplimiento. En efecto, una institución financiera estatal que opera con tasas de interés que no cubren el costo, no es sostenible; el deudor sabe esto y actúa incumpliendo, pues finalmente la institución desaparecerá. A este incentivo al incumplimiento se suma la política de condonar deudas en tiempos electorales, para bosquejar el fracaso de los programas de financiamiento público a tasas subsidiadas.

Villafani y Gonzalez (2006) resumen en cinco puntos las críticas de OSU, que ellos llaman deficiencias estructurales: problemas de agencia (agente-principal); problemas de riesgo moral; dificultades de hacer valer contratos (contract enforcement) poca credibilidad y factibilidad de ejecutar garantías y, finalmente, 
ineficiencias financieras de un intermediario que calcula las rentas políticas de sus acciones.

\section{Inhibición del desarrollo del financiamiento privado}

La política de crédito subsidiado implica de hecho una barrera para la entrada de nuevos oferentes privados quienes no están interesados en entregar subsidios, pues su conducta se rige por criterios de rentabilidad. En este sentido la presencia del estado inhibe el desarrollo del sector financiero privado. De hecho, se afirma que aún muchos años después de haber sido eliminadas, la sedimentación de estás políticas podrían tener efectos perniciosos para el desarrollo del financiamiento de mercado (Villafanni y Gonzalez (2006), para el caso de México). Estos autores afirman: "Esta investigación concluye que, en la mayoría de los casos, el efecto de las intervenciones del Estado en los mercados financieros rurales no sólo ha postergado el surgimiento de iniciativas genuinamente privadas sino que, además, las ha confinado a los espacios en los que la presencia estatal es menor o no existe. Se ha dado así una compleja auto-profesía, donde el Estado justifica las intervenciones por la ausencia del sector privado y donde el sector privado se inhibe de participar por la acción desplazante (crowding out) del Estado, aparentemente justificando, con esto, nuevas intervenciones."(pp.8)

\section{Instituciones Públicas ineficientes}

En general la crítica de OSU en torno al financiamiento público señala que dado que las financieras públicas operan con subsidio no pueden ser eficientes, pues la competencia y la búsqueda de ganancia no los obliga a serlo. Como usualmente se asignan cajones de crédito, estos no se fijan en base a criterios de rentabilidad sino a criterios políticos y se orientan a sectores específicos. Lo cual les impide una adecuada diversificación de la cartera, lo que incrementa su riesgo.

El crédito estatal, desde esta perspectiva, genera incentivos que inducen al riesgo moral y adicionalmente limita su capacidad de diversificación, como resultado su cartera vencida de las Financieras del Estado será alta. Pero quizá más importante, al depender de los recursos fiscales la continuación de estos programas de financiamiento público subsidiado estarán a merced de los vaivenes presupuestales; su mantenimiento depende de la voluntad y capacidad del gobierno y no de la de los participantes en el contrato de crédito: no son sostenibles.

\section{III- LAS MICROFINANZAS Y EL SUBSIDIO}

La intensión de las Instituciones de Microfinanzas (IMF) es proporcionar servicios financieros a las micronegocios y, con ello, ayudar a sus propietarios a salir de la pobreza. Las IMF han adquirido renombre por su capacidad de prestar a aquellos pequeños negocios que los sistemas de crédito tradicionales no pueden, 
pues en general por que este tipo de negocios carecen de garantías e historial crediticio.

Dado que para serlo, las IMF deben ser privadas (con fines de lucro o no, pero privadas) puede considerarse que el movimiento de las microfinanzas es una política de "tercera vía" (Schreiner and Morduch, 2002) una política de mercado para enfrentar un problema social. Adicionalmente, se espera que las IMF sean sostenibles, es decir, que sean capaces de financiar su operación con los ingresos generados por su actividad, para ello se requiere que puedan recuperar adecuadamente su cartera de crédito; que las tasas de interés que ofrezcan reflejen el costo de proveer el servicio. En estas condiciones una IMF no estará distorsionando las señales de mercado al ofrecer crédito, ni racionando a sectores no privilegiados. Aún más, a diferencia del financiamiento público, al no ser ofrecido mediante criterios políticos, el sector racionado por las financieras del gobierno no se verá forzado a acudir a usureros que cobran tasas de interés excesivamente altas. Las IMF competirán con los usureros y prestamistas locales ofreciendo menores tasas de interés que estos.

Ahora bien, la mayoría de las IMF, especialmente las pequeñas, operan con subsidios. "El microfinanciamiento está guiado por el subsidio. Sin él, las microfinanzas serían desconocidas" (Von Pischke, 2007, p.139). Estos subsidios en general provienen de donaciones de organizaciones filantrópicas privadas. Pero aún cuando se obtenga del propio sector privado, viola las condiciones de eficiencia que se asumen vienen como resultado de operar bajo disciplina de mercado.

La argumentación Armendáriz y Morduch (2007) para explicar la utilidad del subsidio a las IMF, vía donaciones de fundaciones u organismos internacionales, se fundamenta en dos promesas de las microfinanzas: que las IMF pueden ser rentables y, segunda, que generan beneficios críticos para aquellos prestatarios que de otra forma no tendrían acceso al crédito. Dado que se espera que en el largo plazo las IMF sean rentables, el subsidio es observado como una opción de corto plazo que permite ofrecer mayores beneficios a través del crédito, expresados también en menores tasas de interés, para la población que se desea apoyar.

El Estado puede verse interesado en el microcrédito como un instrumento para ofrecer servicios de crédito a los microempresarios y, en consecuencia, estar dispuesto a otorgar subsidios a las IMF. Puede, como en el caso que nos ocupa, ofrecer, en vez de subsidio directo, crédito a las IMF que lo demanden y cuyas condiciones resulten favorables.

Sin embargo, Gonzáles (1998) afirma: "Cuando, en contraste, una ONG tiene acceso fácil a fondos públicos, más allá de sus legítimas aspiraciones y, sobre 
todo, más allá de su capacidad de manejarlos a la vez eficiente y creativamente, el acceso fácil a los fondos mata los incentivos a resolver los problemas, si no es que esos fondos son desperdiciados por ausencia de capacidad gerencial para administrarlos sostenidamente."(p.13)

Si el gobierno considera que las IMF tienen una capacidad gerencial adecuada, el ofrecerles dinero "fácil" debe ser una respuesta a la presencia de problemas de información: el mercado no reconoce la rentabilidad potencial de las IMF y les raciona el crédito (tipo red linning); esto es, que algunas IMF sufrirían el mismo problema que sus clientes los microempresarios: la falta de garantías e información sobre estas IMF impide que reciban crédito en condiciones similares a las que ofrecen las instituciones del sistema financiero formal. El gobierno puede tomar el riesgo de prestar a las IMF racionadas por el sistema financiero.

Si la tasa de interés a la que presta el gobierno a estas IMF antes racionadas es menor a la que correspondería si se considera el riesgo, entonces el gobierno estaría canalizando un subsidio. Considerando que se espera que una IMF sea rentable, la política expresada en la intensión de dispersar el crédito público a través de IMF puede evaluarse como una política de corto plazo orientada a impulsar la creación y consolidación de IMF que ofrezcan microcrédito a aquellos pequeños negocios (formales o informales) que lo requieran. Se espera que el subsidio implícito en este financiamiento a las IMF resulte en menores tasas y en más micronegocios apoyados; tal y como se piensa que operan los subsidios.

\section{IV.- EL PAPEL DEL GOBIERNO EN EL FOMENTO DE LAS INSTITU- CIONES DE MICROFINANCIAMIENTO}

A partir de las experiencias derivadas por la implementación del microcrédito se plantean una serie de cuestionamientos sobre el papel del estado en lo que respecta al sector microfinanzas.

Actualmente el debate se centra sobre la función del gobierno en la creación de un entorno favorable para el desarrollo de las IMF. En este sentido resulta importante destacar dos aspectos: el papel del gobierno en relación con el entorno macroeconómico y su responsabilidad en materia de regulación y supervisión de los crecientes mercados de microfinanzas (Foschiato y Stumpo, 2006). A este respecto no existen dudas en cuánto a la función que corresponde al gobierno; en cambio han surgido distintos puntos de vista acerca del papel del estado como promotor, y en algunos casos otorgando financiamiento, del nuevo sector microfinanciero.

Con el crecimiento en el número de IMF este debate ha adquirido relevancia, 
existiendo varios argumentos para la intervención del gobierno como promotor y financiador. El primero de refiere a las características de las entidades financieras, las cuales pueden constituir un bien público debido a que su población objetivo la constituyen individuos y/o grupos sociales rechazados ${ }^{2}$ por el sistema financiero formal (Besley, 1994)ESTA CITADO. Como un bien publico, las innovaciones en microfinanzas pueden beneficiarse de los donadores e inversiones del gobierno para construir una estructura de mayor alcance, sustentabilidad e impacto para las IMF. Según otro argumento, la participación del gobierno a través de subsidios a IMF permite la canalización y focalización de subsidios a la población de bajos ingresos ${ }^{3}$ (Carreón et al, 2006). Este enfoque parte de la efectividad de las IMF para disminuir la pobreza o mejorar los niveles de bienestar de la sociedad.

Bajo el enfoque social las IMF representan para el gobierno un instrumento estratégico para cerrar la brecha entre el sistema financiero formal y los prestamistas informales. Sin embargo, aún no se ha demostrado que el poder de mercado de los prestamistas informales sea socialmente ineficiente, aunque la actuación de estos origine consecuencias negativas para la pobreza, esto debido a que proveen de recursos a sectores no atendidos por el sistema tradicional.

Por otra parte Morduch (1999) menciona que tan solo el 1\% de los programas de microfinanciamiento administrados por las Organizaciones No Gubernamentales (ONG'S) en el mundo son sostenibles financieramente, es decir, estos programas sobreviven gracias a donaciones de fundaciones. Ante esta situación Carreón (2006) menciona: "si el subsidio llega a quien debe llegar, entonces la preocupación debe centrarse en qué tan efectivo está siendo este subsidio desde el punto de vista social. Es decir, en todo caso hay que evaluar el impacto social del subsidio: qué tan efectivos son en incrementar el bienestar de los beneficiarios". pp 63.

Aunado a lo anterior los mercados financieros enfrentan asimetrías en la información debido a las características del intercambio: el dinero es entregado hoy a cambio de una promesa de pago en el futuro. Tal promesa frecuentemente no se cumple, y las instituciones financieras tienen que hacer frente a las consecuencias de esta acción. Las IMF están sujetas a los riesgos derivados del comportamiento de los clientes (riesgo moral), las dificultades en la selección de los deudores (selección adversa) y a los problemas de falta de garantías y seguros.

Cabe mencionar que el gobierno enfrenta los mismos problemas de asimetría en la información como el sector privado, además de no contar con mejores

\footnotetext{
${ }^{2}$ Este rechazo es propiciado por el alto nivel de riesgo que representan y los altos costos que esto conlleva.

${ }^{3}$ Esta postura contrasta con el enfoque de la viabilidad financiera de las IMF.
} 
incentivos que fomenten el pago de la deuda (Besley, 1994). Sin embargo, la intervención del gobierno puede incrementar la eficiencia otorgando garantías y mejorando el acceso a seguros que permitan recuperar los créditos y por ende reactivar el crédito. (Besley, 1994)

Aunque los fallos en el mercado representan una razón para la intervención del gobierno no es la única. Uno de los objetivos de los programas gubernamentales es la mejora del bienestar de la sociedad ${ }^{4}$. Los mercados financieros competitivos pueden distribuir capital a través de instrumentos socialmente inaceptables ${ }^{5}$. Las acciones del gobierno deben proteger y ayudar a la población vulnerable. Las IMF's deben desarrollarse bajo este esquema de apoyo a la población excluida inhibiendo el crecimiento de los prestadores informales. El objetivo del microfinanciamiento es incrementar los ingresos y mejorar el consumo de la población vulnerable. Además existen dos elementos que justifican la intervención del gobierno para proveer fondos: la innovación en los programas de financiamiento y su cobertura nacional. Por tal razón las microfinancieras representan un instrumento de política pública para responder a requerimiento particulares en la provisión de créditos en áreas rurales no atendidas por el sistema formal y para mejorar la situación de pobreza que presenta cierto sector de la sociedad.

El fomento de este nuevo sector financiero es un reto importante para el sector público y requiere de capacidades específicas. En este sentido es necesario diseñar y analizar esquemas de financiamiento público a IMF adaptados a las características específicas del mercado microfinanciero; así como también implantar mecanismos de regulación y supervisión que garanticen el logro de los objetivos sociales.

\section{El microcrédito en México: Programa Nacional de Financiamiento al Microempresario (PRONAFIM).}

En el contexto nacional, la implementación del Programa Nacional de Financiamiento al Microempresario (PRONAFIM) constituyó una experiencia novedosa como programa público para el combate contra la pobreza y la exclusión de las actividades económicas de varios sectores de la población, tanto por su oferta de financiamiento público a IMF's, como por su propósito de brindar servicios financieros y asistencia técnica con una visión integral de las necesidades de la sociedad.

El PRONAFIM surge en el año 2001, como parte de una estrategia, del gobierno federal, orientada a reducir la desigualdad y promover la inclusión en el

${ }^{4}$ El bienestar social justifica la intervención del gobierno aún si no existiera fallos en el mercado.

${ }^{5}$ Como el caso de las instituciones de microcrédito. 
desarrollo económico de sectores productivos y/o sociales tradicionalmente excluidos. Tiene como misión impulsar y fomentar un sistema de instituciones de microfinanciamiento e intermediarios por medio de servicios financieros y no financieros que permitan a estas instituciones consolidar expandir sus labores de crédito, vincularse con otras instituciones y programas de microfinanzas, consolidar y mejorar su desarrollo organizacional, afianzar aspectos normativos de su trabajo de tal forma que se permita apoyar al mayor número de las iniciativas productivas de individuos y grupos sociales que no tienen acceso al crédito a través del sistema financiero tradicional.

Este programa es administrado a través del Fideicomiso del Programa Nacional de Financiamiento al Microempresario (FINAFIM) donde participan la Secretaría de Hacienda y Crédito Público, en su carácter de Fideicomitente Único de la Administración Pública Federal Centralizada, y como Fiduciaria Nacional Financiera, S.N.C.

El PRONAFIM tiene como objetivos generales ${ }^{6}$ :

- Contribuir al establecimiento y consolidación del Sector Microfinanciero para que la población objetivo pueda mejorar sus condiciones de vida, mediante la operación de pequeños proyectos productivos y de negocio;

- Crear oportunidades de autoempleo y de generación de ingresos entre la población de bajos ingresos del país que tengan iniciativas productivas.

A través del primer objetivo se pretende constituir un Sistema de Instituciones de Microfinanciamiento (SIM). Este sistema permitirá atender las fallas del mercado ${ }^{7}$, que actualmente existen en el sistema crediticio nacional, de tal forma que la población objetivo principal pueda acceder continuamente y con base en sus propios méritos, al crédito necesario para llevar a cabo su actividad. Por otra parte el mercado de crédito para actividades productivas, no se encuentra suficientemente desarrollado como para ofrecer apoyo a los diversos segmentos del mercado, incluyendo por supuesto a las personas en condiciones de pobreza, en condiciones de riesgo y viabilidad aceptables.

Para Carreón (2006) el SIM permitirá disminuir los riesgos para las IMF al mejorar la información y reducir los costos de transacción y ofrecerá opciones de financiamiento más eficientes y de riesgo calculado para que las personas en

\footnotetext{
${ }^{6}$ De acuerdo a las Reglas de Operación publicadas en el Diario Oficial de la Federación el día 17 de febrero de 2006.

${ }^{7}$ Mismas que propician el racionamiento del crédito.
} 
condiciones de pobreza y que tengan (o potencialmente puedan tener) proyectos productivos viables, puedan acceder a las ventajas del sistema (que no es sólo crédito, sino información, cálculo y riesgo compartido, así como ventanas de oportunidad de negocios).

El segundo objetivo pretende generar las oportunidades estructurales o sistémicas de mercado (negocio) para que la población en condiciones de pobreza pueda salir de tal condición a través de obtener financiamiento y asistencia técnica para sus proyectos productivos.

La aportación del PRONAFIM ha sido el fortalecimiento general del sector microfinanzas, a través del acercamiento recíproco entre el sector financiero tradicional y las instituciones de microfinanzas. Esto ha permitido la ampliación de servicios financieros incluyentes, a precios razonables, para las personas en situación de pobreza ${ }^{8}$. En el gráfico 1 se muestra el crecimiento que ha tenido el número de microfinancieras incorporadas al programa. De 2001 al 2006 en promedio se incorporaron anualmente 15 nuevas microfinancieras al programa, siendo el año de 2002 el de mayor incidencia con 33 microfinancieras.

\section{Gráfico 1.- Número de microfinancieras incorporadas}

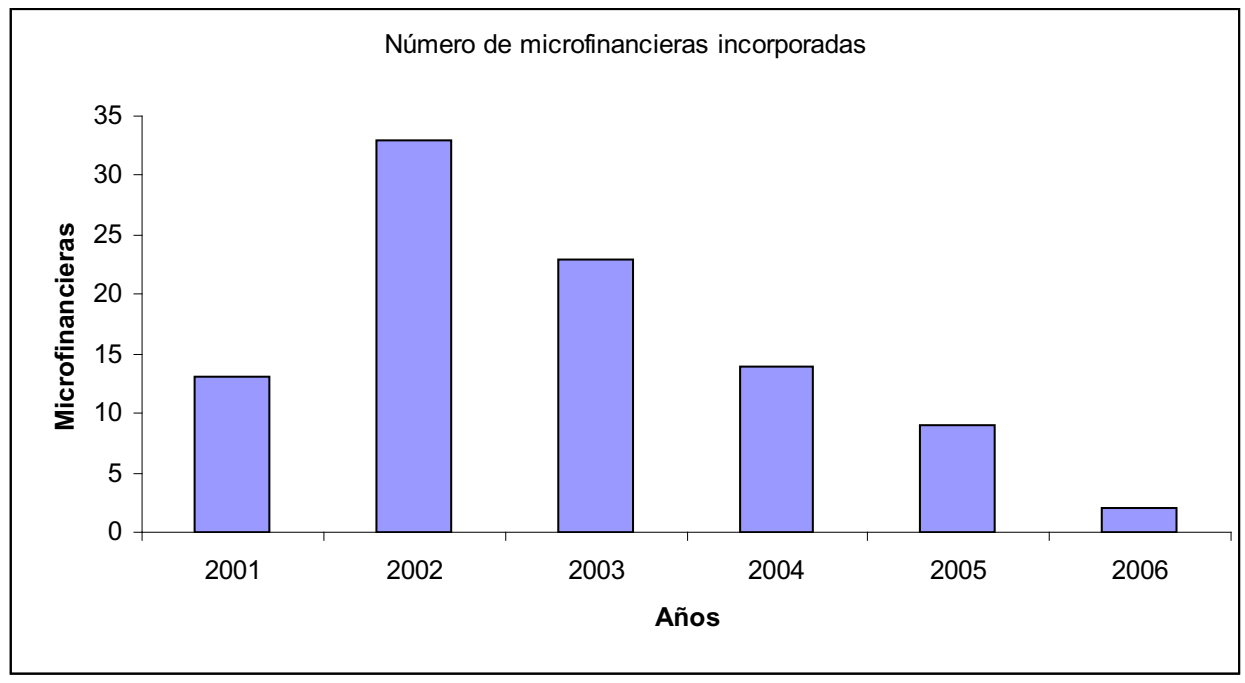

Fuente: Fideicomiso del Programa Nacional de Financiamiento al Microempresario.

${ }^{8}$ En comparación al sector financiero informal. 
Durante el 2006 se incorporaron al programa las IMF's: Desarrollo Empresarial del Campo Mexicano, S. A. de C. V. (DECAMSA), Financiera Finsol, S. A. de C. V. (FINSOL) EUROKASOL, S. A. de C. V. (EUROKASOLI), Finanzas y Servicios Corporativos, S. A. de C. V. (FINSECO), Servicios para el Desarrollo Comunitario, S. A. de C. V. (SERVICOMÚN) y Te Creemos, S. A. de C. V. (TE CREEMOS).

Debido a las características del sector y al alto costo/beneficio ${ }^{9}$ algunas entidades incorporadas al programa no lograron sostenerse en el mercado, tal y como se muestra en el gráfico 2, del $2001 \mathrm{al}$ 2006, en promedio las microfinancieras que se incorporaron anualmente que lograron permanecen en el mercado fue del $55 \%$ y la tasa de mortandad, para las microfinancieras fue del $45 \%$.

\section{Gráfico 2.- Número de microfinancieras activas}

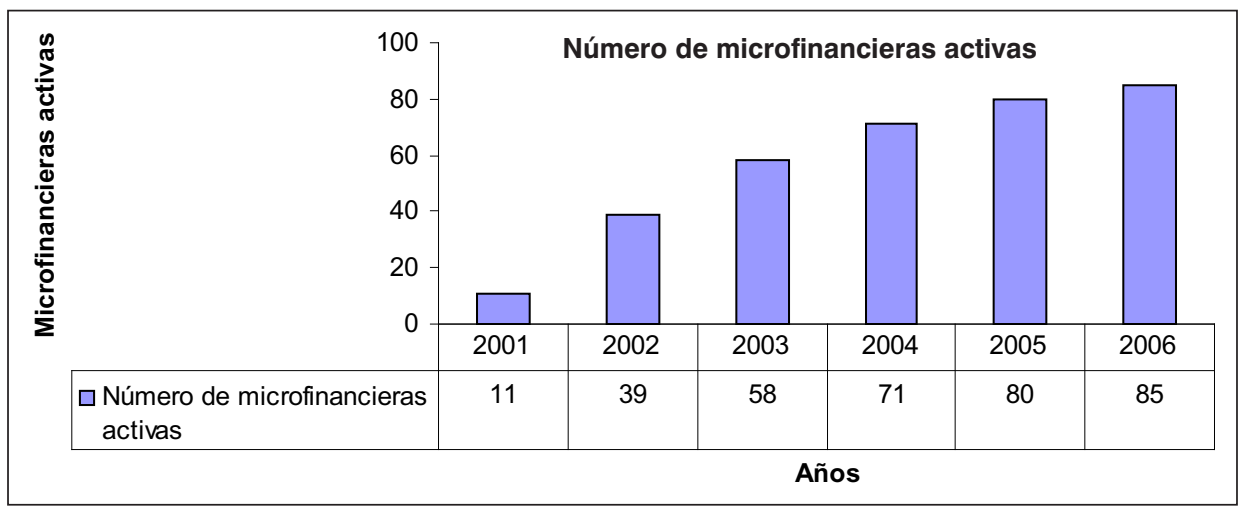

Fuente: Fideicomiso del Programa Nacional de Financiamiento al Microempresario.

En cuánto a las líneas de crédito autorizadas y dispuestas para su uso en el financiamiento en la población objetivo en promedio de 2001 a 2006 el $88 \%$ del monto autorizado fue ejercido; esto implica un alto nivel de penetración en aquellas zonas marginas del país (Ver gráfico 3). Por lo que respecta al monto colocado en el período 2001-2006 ascendió a 1,081 millones de pesos; resultando el año 2004 el de mayor colocación con 327 millones de pesos. Además durante el mismo período se otorgaron 1,019,491 microcréditos lo que correspondió a la cantidad de $\$ 4,564$ millones de pesos.

\footnotetext{
${ }^{9}$ Fuente: Boletín de PRONAFIM correspondiente a marzo 2006.
} 
Gráfico 3.-Líneas de Crédito

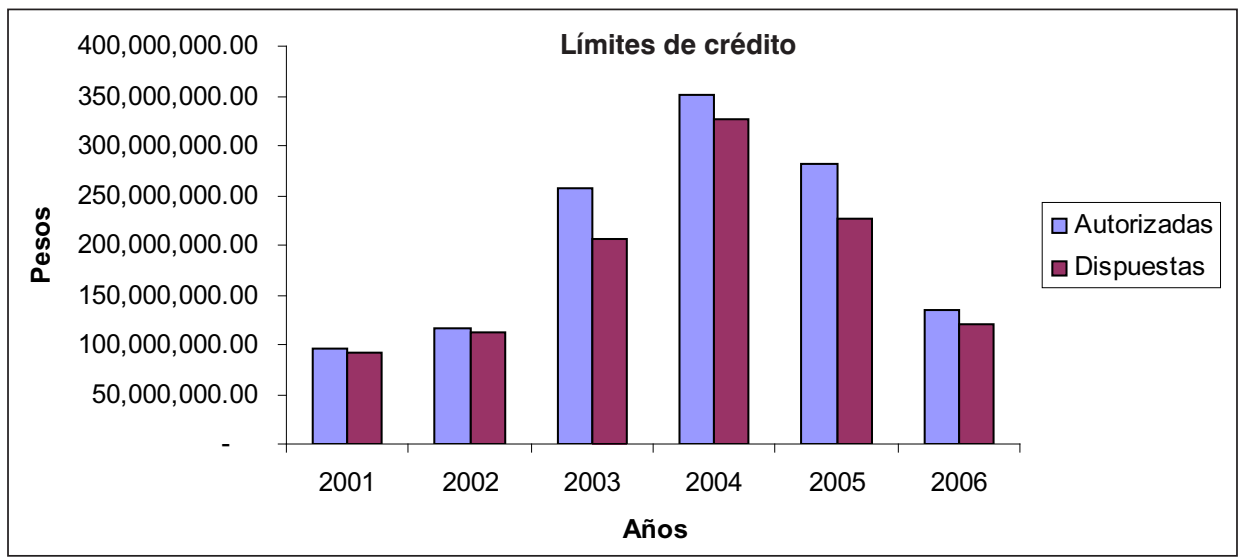

Fuente: Fideicomiso del Programa Nacional de Financiamiento al Microempresario.

En cuánto a la cobertura del programa en el 2006, el gráfico número 4 nos muestra que el estado con mayor número de créditos otorgados fue el Estado de México (35.1\%), seguido del Distrito Federal (13.9\%) y Chiapas (7.0\%). Por su parte, las entidades con menor porcentaje de créditos otorgados son Quintana Roo, Aguascalientes y Durango. Esto nos da una alta concentración de los créditos en el Centro del país, donde el Estado de México y el D. F. tienen casi el 50\% del total. Por el contrario, existen algunos estados en los cuales la participación es casi nula.

A pesar de los resultados obtenidos durante sus años de operación el segundo objetivo general no ha logrado cumplirse, en su totalidad, debido a que los procesos de operación, supervisión y evaluación están particularmente dirigidos a consolidar a las instituciones de microfinanciamiento. Esto no significa que el programa en su conjunto no pueda cumplir con este objetivo, sino que el diseño y estructura de sus procesos no evidencian un impacto directo sobre el mismo. Para Carreón (2006) en parte, esto encuentra explicación en el hecho que el PRONAFIM consiste en un programa de intermediación, que pretende alcanzar a la población objetivo a través de las IMF.

\section{V.- ELEMENTOS DE EVALUACIÓN DE LA POLÍTICA}

De la revisión de los planteamientos del movimiento de las microfinanzas se desprenden los siguientes criterios de análisis: eficiencia, sostenibilidad (cumplimiento y tasas que recuperen los costos de proporcionar el crédito), redistribución del ingreso y democratización del crédito, fomento del sector privado e instituciones eficientes, menores tasas y más IMF. 
Gráfico 4.- Proporción de créditos otorgados

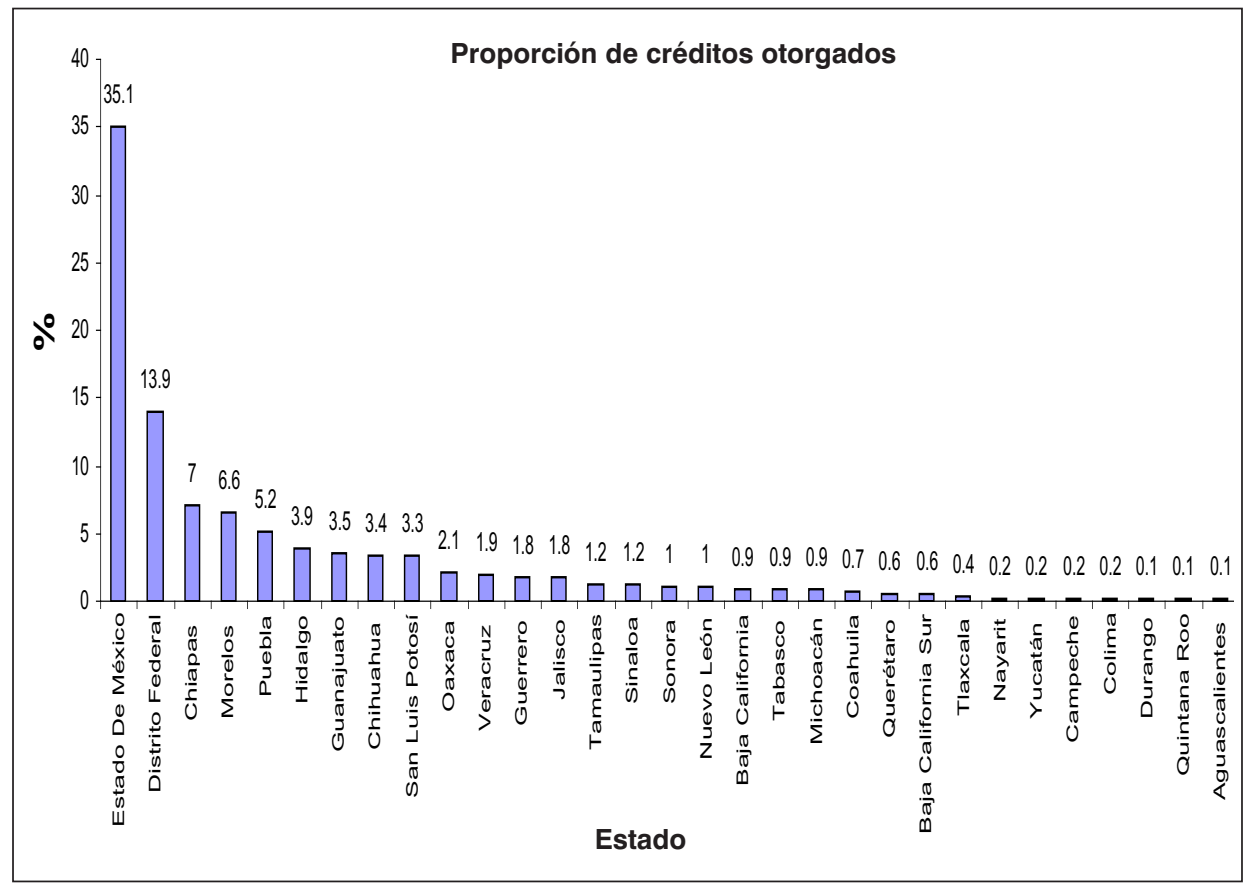

Fuente: Fideicomiso del Programa Nacional de Financiamiento al Microempresario.

Algunas organizaciones de microcrédito asumieron la figura jurídica de Sociedades Financieras de Objeto limitado (SOFOL). Este tipo de organización, que fue sustituido por otras figuras a partir del 2007, exigía la especialización del intermediario financiero en un solo tipo de operación financiera. Así los informes en cuanto a ingresos gastos y patrimonio que estas organizaciones produjeron están exclusivamente vinculados con la oferta de crédito, esto hace posible evaluar las fuentes de ingreso y los costos de ofrecer crédito en los diferentes mercados donde las SOFOLES operaron. La comisión Nacional Bancaria y de Valores (www.cnbv.gob.mx -sociedades financieras de objeto limitado) publicó los estados financieros (resultados y balance) de estas SOFOLES correspondientes al periodo 2002 - 2006. Con estos datos formamos el siguiente cuadro. 


\section{Cuadro 1.- SOFOLES}

\section{Promedio de datos anuales periodos 2002 al 2006}

\begin{tabular}{|l|c|c|c|c|c|c|}
\hline & $\begin{array}{c}\text { TOTAL DE } \\
\text { PRÉSTAMOS } \\
\text { TOTAL DE } \\
\text { RECURSOS }\end{array}$ & $\begin{array}{c}\text { PRESTAMOS } \\
\text { DEL GOBIERNO } \\
\text { TOTAL DE } \\
\text { RECURSOS }\end{array}$ & $\begin{array}{c}\text { PRESTAMOS } \\
\text { DEL GOBIERNO } \\
\text { TOTAL } \\
\text { PRESTAMOS }\end{array}$ & $\begin{array}{c}\text { intereses } \\
\text { cobrados } \\
\text { cartera de } \\
\text { crédito } \\
\text { vigente }\end{array}$ & $\begin{array}{c}\text { intereses } \\
\text { pagados } \\
\text { deuda }\end{array}$ & $\begin{array}{c}\text { margen } \\
\text { implícito } \\
\text { de inter- } \\
\text { mediación }\end{array}$ \\
\hline $\begin{array}{l}\text { agrícolas y de educación } \\
\text { (totalmente financiadas } \\
\text { por el gobierno) (1) }\end{array}$ & $73.5 \%$ & $71.8 \%$ & $97.7 \%$ & $6.1 \%$ & $2.3 \%$ & $3.8 \%$ \\
\hline $\begin{array}{l}\text { microcrédito y crédito al } \\
\text { consumo con financiamiento } \\
\text { privado y sin financiamiento }\end{array}$ & & & & & & \\
público (2) & $41.4 \%$ & $0.0 \%$ & $0.0 \%$ & $46.6 \%$ & $4.2 \%$ & $42.3 \%$ \\
\hline $\begin{array}{l}\text { microcrédito grupal con } \\
\text { financiamiento mixto (3) } \\
\text { crédito automotriz(4) } \\
\text { crédito hipotecario (5) }\end{array}$ & $72.8 \%$ & $34.0 \%$ & $42.4 \%$ & $51.6 \%$ & $8.0 \%$ & $43.7 \%$ \\
\hline
\end{tabular}

(1) SOCIEDAD AGROINDUSTRIAL SOFIHAA, SOFOL; AGROFINANCIERA DEL NOROESTE, SOFOL; AGROFINANZAS, SOFOL; AGROPECUARIA FINANCIERA, SOFOL; SOC DE FOMENTO A LA EDUCAC SUP, SOFOL.

(2) FINANCIERA ALCANZA, SOFOL; CETELEM MEXICO SOFOL; CREDITO IDEAL, SOFOL (antes CREDITO PROGRESO); UNIMEX FINANCIERA, SOFOL; CAPITAL PLUS, SOFOL; FINANCIERA TU ELIGES, SOFOL; CREDITO FAMILIAR, SOFOL (Antes:Servs.de Cred.AsSOc); FINANCIERA INDEPENDENCIA, SOFOL; CREDITOS PRONEGOCIO, SOFOL.

(3) FINANCIERA FINSOL, SOFOL; FINANCIERA COMPARTAMOS, SOFOL.

(4) NR FINANCE MEXICO, SOFOL; FORD CREDIT DE MEXICO, SOFOL; GMAC FINANCIERA, SOFOL; SERVICIOS FINANCIEROS NAVISTAR, SOFOL.

(5) CREDITO INMOBILIARIO, SOFOL; HIPOTECARIA CREDITO Y CASA, SOFOL; FINCASA HIPOTECARIA, SOFOL; HIPOTECARIA NACIONAL, SOFOL; GE MONEY CREDITO HIP., SOFOL (Antes: General Hipot); METROFINANCIERA, SOFOL; ING HIPOTECARIA, SOFOL; PATRIMONIO, SOFOL; HIPOTECARIA SU CASITA, SOFOL.

Fuente: Elaboración propia en base a datos de la comisión Nacional Bancaria y de valores (www.cnbv.gob.mx -sociedades financieras de objeto limitado).

\section{Sostenibilidad (cumplimiento y tasas que recuperen los costos de proporcionar el crédito)}

En nuestro cuadro se observa que en el caso del grupo 1, en que la deuda total de la sofol se contrató con organizaciones gubernamentales, en efecto las tasas son sensiblemente más bajas que las otras financieras en la muestra. En este grupo se encuentran dos sofoles con actividad dirigida al campo y una orientada a financiar estudios superiores. Las actividades que financian los intermediarios de este grupo pueden considerarse de carácter "social”, esto sugiere que el gobierno al otorgar este financiamiento está dispuesto a perder "eficiencia", subsidiando dichas actividades mediante crédito a bajo costo (sin cobrar por riesgo) pues considera estos sectores de interés general. 
Sin embargo, el alto nivel de apalancamiento que presentan estas organizaciones (en el caso de laa SOFOLES dedicadas a préstamos para educación y agricultura el $97.7 \%$ de sus recursos provienen de crédito público) el gobierno sugiere que se podría estar creando incentivos al sobreendeudamiento y una actitud de riesgo moral. En efecto, el acceso a crédito de bajo costo abre la posibilidad de que la empresa que reciba este tipo de crédito adopte un comportamiento de riesgo moral. Dicho riesgo es trasladado al gobierno (que adicionalmente no cobra por solvertar esta situación); si los proyectos son exitosos la IMF la optará por cubrir su deuda con el gobierno con lo que, de cierta forma, los propietarios de la IMF "recuperan" sus activos y realizan altas ganancias; en caso contrario, dado que los proyectos habrían fallado, los propietarios de la IMF optarían por no pagar la deuda y retirarse pues de hecho la IMF sería insolvente. Así, desde el momento que los recursos propios son como una opción de compra en los activos de la empresa, los accionistas tienen incentivos, como propietarios, a invertir en proyectos de alto riesgo..." (Marín y Rubio, 2001)

Considerando esta posibilidad las sofoles del grupo 1, a las que podemos llamar dispersoras del crédito público podrían presentar problemas de insostenibilidad (por depender del subsidio) y de incumplimiento (que se podrían estar originando desde la conducta maximizadora de la institución). Esto pudo haber ocurrido con aquellas microfinancieras incorporadas al PRONAFIM que no lograron sobrevivir.

Debemos, sin embargo, ser precavidos pues deberá estudiarse de manera más detallada conociendo las condiciones concretas de la tecnología del crédito que cada financiera aplica y los incentivos específicos en cada caso para el cumplimiento. Estas organizaciones podrían optar por establecer una relación de largo plazo con el estado y evitar incurrir en sobre endeudamiento de alto riesgo. De ser este el caso, el gobierno estaría aplicando una política de amortiguamiento del racionamiento del crédito que sufrirían estas instituciones. En este caso el estado debería evaluar la calidad de la cartera de la IMF, hacer creíble el posible retiro del financiamiento y veracidad del cobro a los propietarios de la IMF.

\section{Alcance e impacto}

Si bien el uso de las IMF como instrumento de dispersión del crédito público opera como política de promoción a las propias IMF, en términos de política de desarrollo este debe ser considerado un objetivo intermedio pues el objetivo final, tal como en el caso PRONAFIM, es impulsar la creación y consolidación de micronegocios. Se asume entonces que la actividad de las IMF es eficaz en el fomento y desarrollo de las microempresas, aunque esto aún no se ha demostrado ya que el desarrollo de proyectos productivos depende de otros factores como lo son la capacitación, el mercado y la experiencia del emprendedor. 
Los efectos del microcrédito en este tipo de negocios, usualmente informales, es difícil de cuantificar ${ }^{10}$. Esta dificultad surge de la naturaleza de los micronegocios: se trata de pequeños emprendimientos que involucran el autoempleo o a empresas familiares, en ellos los recursos de la familia y los del micronegocio se mezclan impidiendo cuantificar el impacto en los propios micronegocios o en el bienestar de la familia (Adams, 1987). Esta circunstancia también hace nebulosa e innecesaria la diferenciación entre créditos al consumo a pobres y microcrédito.

Ahora bien, si se asume, como hacen los partidarios de las microfinanzas, que cualquier transacción en el mercado se realiza sólo porque reporta beneficios a los participantes, entonces un microcrédito otorgado, por altas que sean las tasas de interés, genera beneficios a los microempresarios. Bajo este supuesto las microfinanzas se han propuesto como su principal objetivo tener cada vez un mayor alcance, o desde su perspectiva, beneficiar a un mayor número de micronegocios con el crédito. En este sentido, la política de fomento al microcrédito puede verse como una política que se suma a esta visión y se propone expandir el alcance de las microfinanzas.

Sin embargo, en términos de alcance, el crecimiento del microcrédito en México ha sido acelerado ${ }^{11}$. Ésta se concentra en unas cuantas instituciones ${ }^{12}$ como son Banco Compartamos (con más de un millón de clientes), Financiera Independencia (con 833,902 clientes), Caja Popular Mexicana (765,056 clientes) y Caja Libertad $(326,107)$. Esto podría ser una señal de que el sector microcrédito no requiere de apoyo público para crecer.

\section{Eficiencia y fomento a la competencia}

Si bien el objetivo de expandir el alcance de las microfinanzas parece no requerir del apoyo estatal, lo concentrado del mercado y los descomunales márgenes de intermediación que estas IMF suelen aplicar (de acuerdo a la CNBV las tasas de interés fijadas, en general, son cercanas al 100\%) pueden considerarse un argumento a favor de que el gobierno intervenga fomentando la competencia. La política de promoción a las IMF mediante el uso de estas como dispersoras del crédito público, puede ser interpretada como una política de corto plazo para incentivar la competencia e incrementar tanto la eficiencia como el alcance.

El incremento de la competencia aparece como condición indispensable para que un mayor alcance logre efectivamente la eficiencia y los beneficios ofrecidos por la teoría del mercado que da soporte a la microfinanzas. El argumento de que

\footnotetext{
${ }^{10}$ Esto debido a su naturaleza no formal.

${ }^{11}$ De acuerdo a The Mix en el período comprendido entre 2006 y 2008 la tasa de crecimiento de este sector fue del $35 \%$.

${ }^{12}$ Fuente: The Mix.
} 
la eficiencia se logra cuando la asignación del crédito a los pequeños negocios se rige con criterios de mercado exige, entre otras cosas, que la competencia sea lo suficientemente amplia.

Como puede observarse en el cuadro 1 los márgenes de intermediación del grupo 2 (instituciones de microcrédito que no reciben financiamiento del estado pero que gozan de amplio financiamiento privado) son, en términos comparativos, muy altos. Esto puede indicar que la competencia en el sector aún no es lo suficientemente desarrollada y que el crédito público podría jugar un papel importante compartiendo riesgo e incrementando la competencia. Con programas como el arriba descrito el gobierno promovería una mayor cobertura al incrementar el número de oferentes, el cual se vería magnificado con el efecto precio resultado de impulsar la competencia.

$\mathrm{Al}$ observar a las Instituciones de microfinanzas del grupo 3 (instituciones de microfinanzas tradicionales -técnica grupal), Finsol, que puede considerarse competidora de Compartamos, contó en este periodo con un considerable apoyo del crédito público ( $59.5 \%$ del total de sus recursos se obtuvieron de crédito del instituciones del gobierno); sus tasas implícitas, tanto la activa como la pasiva, fueron menores que su referido competidor, esto nos puede dar indicios de que el gobierno se propondría, con programas como PROFIN, intensificar la competencia en el mercado del microcrédito con el fin de reducir los márgenes de intermediación.

\section{Redistribución del ingreso y democratización del crédito}

Comparemos el grupo 4 (orientado a financiar la compra de automóviles) y el 5 (sofoles que operan en el mercado hipotecario -en su mayoría de vivienda popular). En ambos tipos de crédito el bien financiado opera como garantía, en consecuencia no pueden considerarse microcréditos aún en el caso de que algunos de los prestatarios inmobiliarios pudieran ser considerados pobres.

Las sofoles automotrices prácticamente no recibieron crédito del gobierno y las hipotecarias lo recibieron intensamente (casi el 70\% de los préstamos recibidos). Sus tasas implícitas y su margen de intermediación son muy similares considerando que los plazos del crédito que ofrecen son usualmente diferentes. Debe señalarse que la intensión del gobierno es apoyar programas de vivienda popular por lo que el estado podría estar absorbiendo el margen de riesgo implícito en prestar a las clases populares.

$\mathrm{Al}$ observar las tasas implícitas y los niveles de apalancamiento de Finsol (apoyada con crédito del gobierno) éstos son similares a las de Pronegocio, quien recibe abundante crédito de uno de los más grandes bancos comerciales de México. 
Ciertamente sus tecnologías de crédito son muy diferentes, en general la primera se orienta a microcrédito grupal y la segunda a crédito individual a pymes, pero esta similitud mueve a pensar que quizá el crédito del gobierno podría estar operando como instrumento para que el estado absorba el diferencial de riesgo en estas tecnologías.

Estos indicios permiten levantar la posibilidad de que el estado esté operando como un igualador que permite democratizar las condiciones en que se ofrece crédito. Como el subsidio implícito en la prima de riesgo no cobrada por el estado corresponde al riesgo subyacente en el crédito a los sectores populares, no ocurre el efecto de redistribución negativa ("asignación a favorecidos") señalada por los analistas arriba referidos.

\section{VI.- CONCLUSIONES}

La disertación aquí presentada apunta a que la participación del Estado en el crédito popular, a pesar de las posibles utilizando a las instituciones de crédito popular y de microcrédito como dispersoras del crédito es favorable, es decir, cumple con el objetivo de asignar crédito público evitando el problema de incumplimiento que ha caracterizado al crédito estatal.

El acercamiento aquí presentado al análisis de la política pública de utilizar a las Instituciones de microfinanzas (IMF) como dispersoras del crédito público partió de criterios presentados por la literatura vinculada a las microfinanzas en torno a la participación del estado en el mercado del crédito. Esta revisión nos llevó a los siguientes criterios de evaluación: sostenibilidad, eficiencia y democratización de las condiciones de acceso al crédito.

Para obtener algunos elementos en torno al desempeño de esta política se revisaron los resultados presentados del programa PRONAFIM, así como una muestra de SOFOLES para permitir algunas comparaciones entre diferentes tipos de oferentes de crédito. Con estos elementos se perfilan los siguientes resultados en torno a los criterios elegidos.

Ciertamente, al menos en el plano prospectivo, el ofrecer crédito a sectores de interés social (como los son el sector agrícola y la educación) a través de organizaciones de microfinanzas operando dispersores del crédito público permite crear incentivos al cumplimiento a nivel de los usuarios finales del crédito, los cuales se esperan sean microempresas. Con esto se estaría contrarrestando el viejo problema de incumplimiento que han presentado los usuarios del crédito público. Asimismo, dado que el crédito final se ofrece en condiciones de mercado, se elimina el problema señalado en la literatura de la distribución regresiva del crédito 
público. Estos elementos estarían contribuyendo a la sostenibilidad de las IMF y con ello al éxito del programa.

Sin embargo, los argumentos y los indicios presentados permiten levantar la posibilidad de que algunas de las IMF receptoras de financiamiento público tiendan al sobreendeudamiento y a tomar inversiones excesivamente riesgosas. Asimismo, al no estar claros los criterios conforme a los cuales se seleccionan las microfinancieras que serán beneficiarias del crédito público, se podría presentar ineficiencias al asignar recursos no a las IMF'S que presentan estructuras financieras sólidas que tienen por objetivo canalizar los créditos a la población no atendida por la banca comercial; sino a aquellas que aprovechan situaciones coyunturales para especular con recusos. De confirmarse estas posibilidades, estas circunstancias podrían hacer que las limitaciones del crédito público señaladas en la literatura reseñada se vuelvan a presentar, aunque no a nivel de usuario final del crédito sino a nivel de la institución dispersora del crédito público.

Por ahora no es posible adelantar conclusiones al respecto, sin embargo resulta pertinente señalar que el éxito de la política en cuestión, proyectado en la sostenibilidad del las IMF beneficiadas por el crédito público, dependerá de si programas como el PRONAFIM logra crear incentivos hacia las IMF que las lleven a un manejo prudente de los recursos públicos basado en la rentabilidad de largo plazo.

Las literatura en torno a las microfinanzas plantea que si el crédito se ofrece en condiciones de mercado entonces un mayor alcance, esto es una mayor cobertura entre la población objetivo, debe ser la meta de las microfinanzas. El crecimiento en el alcance de las instituciones de microfinanzas ha sido vertiginoso y este se ha logrado sin una contribución significativa del crédito público. Esto plantea la posibilidad de que la presencia del estado en el mercado del microcrédito sea innecesaria. Sin embargo, este crecimiento se concentra en unas cuantas instituciones y esta concentración puede estar influyendo en el enorme margen de intermediación que dichas instituciones aplican. La información presentada perfila la posibilidad de que la presencia del estado financiando instituciones de microfinanzas pueda contribuir a incrementar la competencia y, en tanto, reducir precios e incrementar la eficiencia, lo cual operaría en beneficio del microempresario.

Al utilizar a las IMF como dispersoras del crédito, el estado tiende a absorber la prima de riesgo correspondiente a prestar a los sectores de menor ingreso. $\mathrm{Si}$ este subsidio se traslada al microempresario a manera de menores tasas de interés, que tiendan a acercarse a las que pagan otros sectores de la economía, el estado estaría impulsando condiciones de acceso al crédito que aliviarían, al menos en el aspecto costo del crédito, las presiones competitivas que sufren los micronegocios. 
Finalmente, esta primera aproximación nos permite señalar que el Estado, al impulsar organizaciones de microcrédito diferentes a las dominantes en el mercado, podría estar operando como detonador de una mayor competencia, la cual podría, no solo estimular una mayor cobertura entre la población objetivo, también podría contribuir a reducir los márgenes de intermediación. Aunque en el caso del PRONAFIM existe una gran concentración del crédito en la zona Centro del país.

\section{BIBLIOGRAFÍA}

1 Adams W, Dale (1987). "El enigma de proyectos de crédito exitosos en mercados financieros fracasados”, en Dale W. Adams, Claudio González V y J.D. Von Pischke (Editores) "Crédito Agrícola y Desarrollo Rural: La Nueva Visión" Ohio State University, Trejos San José de Costa Rica 1987

2 Armendariz de Aghion, Beatriz y Morduch, Jonathan (2005) "The Economics of Microfinance". The MIT Press, Cambridge, Massachusetts, London Ingland, 2005 .

3 Besley, T. (1994). How do market failures justify interventions in rural credit markets?. The World Bank Research Observer.

4 Carreón Rodríguez V et al. (2006). Evaluación de resultados del Programa Nacional de Financiamiento al Microempresario (PRONAFIM) correspondiente al ejercicio fiscal 2006. Centro de Investigación y Docencia Económicas, A.C.

5 CGAP (2004) "Resumen de los principios clave de las microfinanzas" fecha de consulta 27 oct.2007. http://www.cgap.org/portal/site/CGAP/menuitem.64 co3ec40a6d295067808010591010ao/

6 Dale, et all (1987) Adams, Dale W.; González Vega, Claudio; Von Pishke, J.D. (1987) (Editores) "Crédito agrícola y desarrollo rural: la nueva visión" $2^{\circ}$ edición. Ohio State University 1990, págs. 1 - 360

7 Diario Oficial de la Federación. Reglas de Operación del Programa Nacional de Financiamiento al Microempresario.17 de febrero de 2006, p. 34.

8 Charles, J. (2006). “La saga de apoyos financieros”. Boletin de Microfinanzas. Año 2, marzo de 2006. 
9 Foschiattio P. y Stumpo G. (2006). Políticas municipales de microcrédito un instrumento para la dinamización de los sistemas productivos locales: Estudios de caso en América Latina. CEPAL.

10 Galvis, Vicente (1981) "Aspectos teóricos de la política de tasas de interés en países en desarrollo" Serie Ensayos. Centro de Estudios Monetarios Latinoamericanos (CEMLA), México, 1981.

11 González, Claudio (1987). "Comportamiento de los acreedores agropecuarios al racionar el crédito: La ley de hierro de las restricciones a las tasas de interés”, en Dale W. Adams, Claudio González V y J.D. Von Pischke (Editores) "Crédito Agrícola y Desarrollo Rural: La Nueva Visión" Ohio State University, Trejos San José de Costa Rica 1987

12 González Vega, Claudio. (1998) "El papel del Estado en la promoción de los servicios financieros rurales" Rural Finance Program Department of Agricultural, Environmental and Development Economics The Ohio State University. Economics and Sociology Occasional Paper No. 2529 (Págs. 1-16) http:// aede.osu.edu/programs/RuralFinance/PDF\%20Docs/Publications\%20List/ Papers/98Po9.pdf

13 González Vega, Claudio. (1998) "El papel del Estado en la promoción de los servicios financieros rurales" Rural Finance Program Department of Agricultural, Environmental and Development Economics The Ohio State University. Economics and Sociology Occasional Paper No. 2529 (Págs. 1-16) http:// aede.osu.edu/programs/RuralFinance/PDF\%20Docs/Publications\%20List/ Papers/98Po9.pdf

14 Lapenu, C. (2000). "The role of state in promoting microfinance institucions." FNCD Discussion paper núm. 89. International Food Policy Research Institute.

15 Marín, José M y Rubio, Gonzalo (2001) "Economía Financiera" Antoni Bosch, Editor. España, 2001.

16 McKinnon, Ronald (1973) "Dinero y capital en el desarrollo económico" Centro de Estudios Monetarios Latinoamericanos, México D.F. Ed. En español, 1974.

17 Shaw (1973), Edward S. "Financial Deepening in Economic Development", New York. Oxford University Press, 1973. 
18 Schreiner, M and Morduch, J. (2002), "Oportunities and Challenges for Microfinance in the United States Schreiner", en Replicating Microfinance in the United States, Woodrow Wilson center press, Washington, D.C. by Fannie Mae Foundation, 2002, pp. $19-61$.

19 Villafani Ibarnegaray, Marcelo; González Vega, Claudio (2006) "El Estado y las finanzas rurales y populares en México" Proyecto AFIRMA, The Ohio State University.; The Ohio State University. México, Págs. 1-89. Noviembre de 2006 (Mimeo)

20Von Pischke, J.D. "Methodenstreit and sustainability in microfinance: Generalizations describing institutional frameworks" en What's wrong with microfinance? Dichter, Thomas; Harper Malcolm. (Editors) Chap. 12. Practical Action publishing, UK 2007, págs. 137 - 148. 